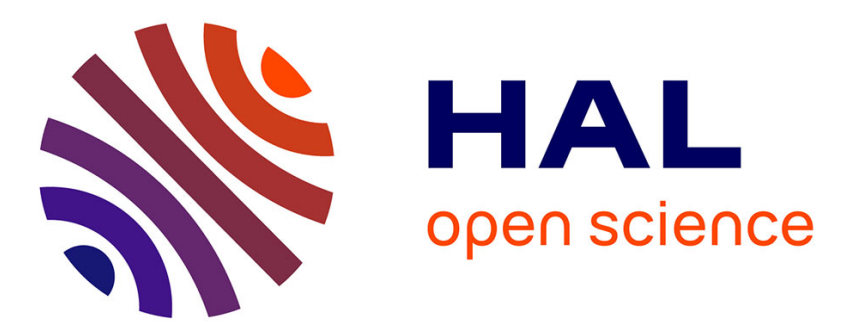

\title{
Micropatterning thermoplasmonic gold nanoarrays to manipulate cell adhesion
}

Min Zhu, Guillaume Baffou, Nikolaus Meyerbröker, Julien Polleux

\section{To cite this version:}

Min Zhu, Guillaume Baffou, Nikolaus Meyerbröker, Julien Polleux. Micropatterning thermoplasmonic gold nanoarrays to manipulate cell adhesion. ACS Nano, 2012, 6 (8), pp.7227-7233. $10.1021 / \mathrm{nn} 302329$ c . hal-00728839

\section{HAL Id: hal-00728839 \\ https://hal.science/hal-00728839}

Submitted on 7 Sep 2012

HAL is a multi-disciplinary open access archive for the deposit and dissemination of scientific research documents, whether they are published or not. The documents may come from teaching and research institutions in France or abroad, or from public or private research centers.
L'archive ouverte pluridisciplinaire HAL, est destinée au dépôt et à la diffusion de documents scientifiques de niveau recherche, publiés ou non, émanant des établissements d'enseignement et de recherche français ou étrangers, des laboratoires publics ou privés. 


\section{Micropatterning Thermoplasmonic Gold Nanoarrays to Manipulate Cell Adhesion}

\begin{tabular}{|r|l|}
\hline Journal: & ACS Nano \\
\hline Manuscript ID: & Draft \\
\hline Manuscript Type: & Article \\
\hline Date Submitted by the Author: & n/a \\
\hline Complete List of Authors: & $\begin{array}{l}\text { Zhu, Min; Chinese Academy of Sciences, State Key Laboratory of High } \\
\text { Performance Ceramics and Superfine Microstructure, Shanghai Institute of } \\
\text { Ceramics } \\
\text { Baffou, Guillaume; Aix-Marseille Université, CNRS, Fresnel Institute, Ecole } \\
\text { Centrale Marseille } \\
\text { Meyerbröker, Nikolaus; Universität Heidelberg, Angewandte Physikalische } \\
\text { Chemie } \\
\text { Polleux, Julien; Max Planck Institute of Biochemistry, Molecular Medicine }\end{array}$ \\
\hline
\end{tabular}

SCHOLARONE $^{\text {'m }}$
Manuscripts 


\title{
Micropatterning Thermoplasmonic Gold Nanoarrays
}

\section{To Manipulate Cell Adhesion}

\author{
Min Zhu ${ }^{1,2}$, Guillaume Baffou ${ }^{3}$, Nikolaus Meyerbröker ${ }^{4}$, Julien Polleux $^{1^{*}}$ \\ ${ }^{1}$ Max Planck Institute of Biochemistry, Department of Molecular Medicine, 82152 Martinsried, \\ Germany \\ 2 State Key Laboratory of High Performance Ceramics and Superfine Microstructure, \\ Shanghai Institute of Ceramics, Chinese Academy of Sciences, 1295 Ding-Xi Road, \\ Shanghai, 200050, PR China \\ ${ }^{3}$ Fresnel Institute, CNRS, Aix-Marseille Université, Ecole Centrale Marseille, Campus de St \\ Jérôme, 13013 Marseille, France \\ ${ }^{4}$ Angewandte Physikalische Chemie, Universität Heidelberg, Im Neuenheimer Feld 253, \\ 69120 Heidelberg, Germany
}

\begin{abstract}
Analysis of cell behavior while reversibly controlling the interactions between the extracellular matrix (ECM) and cell surface receptors such as integrins would allow investigating reciprocal signaling circuits between cells and their surrounding environment. Engineering microstructured culture substrates functionalized with switchable molecules remains the most adopted strategy to trigger surface adhesive properties although these systems exhibit limited reversibility and necessitate sophisticated preparation procedures. Here, we report a straightforward protocol to fabricate biofunctionalized micropatterned gold nanoarrays that favor one-dimensional cell migration and function as plasmonic nanostoves to physically block and orient the formation of new adhesion sites. Being reversible and not restricted spatiotemporally, thermoplasmonic approaches will open new opportunities to further study the complex connections between ECM and cells.
\end{abstract}

KEYWORDS: block copolymer micellar lithography . deep UV lithography . thermoplasmonics . cell adhesion . nanobiotechnology

*Address correspondence to polleux@biochem.mpg.de.

Submitted for review May 26 2012. 
Analysis of cell behavior by exogenously controlling cellular processes with the help of biophysical means represents a complementary approach to classic methods, such as gene knockdown ${ }^{1}$ and pharmacological strategies. ${ }^{2}$ Reversible manipulation of interactions between the extracellular matrix (ECM) and cell surface receptors such as integrins would allow triggering cell adhesion to systematically analyze reciprocal signaling circuits between cells and their surrounding environment. The combination of micro/nanofabrication with stimulus-responsive surface chemistries proved to be a versatile tool to dynamically modify extracellular environments. External stimuli such as voltage and light can switch on or off the adhesive properties of surfaceanchored molecules to dissect new aspects of cell adhesion, ${ }^{3,4}$ detachment $^{5}$ and migration. 6 , 7 However, limitations regarding advanced fabrication procedures, stimulus localization and binding reversibility remain critical to extend the applicability of chip-based devices and transfer them from bioengineering to cell biology laboratories. ${ }^{8}$ To overcome such issues, the emergence of new strategies would be critical to exogenously manipulate cells with the help of biophysical means.

In contrast to non-invasive approaches, variations in culture medium temperature can be used to release cells when cultured onto dishes functionalized with thermo-responsive polymers. ${ }^{9}$ To make use of temperature in a less invasive manner, electrically heated microwire electrodes were utilized to induce cellular release of heat-shock proteins. ${ }^{10}$ Such approaches never attempted to manipulate allosteric interactions between cells and their binding environment due to restricted spatial freedom in generating local heat. Gold nanoparticles can act as heat nanosources when excited at their 
plasmonic resonance due to enhanced light absorption. Thermoplasmonics and related photothermal effects opened a new realm of applications in Nanotechnology, ${ }^{11}$ which enabled to engineer optofluidic systems, ${ }^{12,}{ }^{13}$ analyze DNA dissociation, ${ }^{14}$ destroy selectively cancerous cells ${ }^{15,} 16$ and release intracellularly bioactive molecules. $^{17}$ So far these applications exclusively used dispersed nanoparticles but never immobilized ones, which would allow designing functional platforms to manipulate cell adhesion.

Here, we report a straightforward protocol to fabricate biofunctionalized micropatterned gold nanoarrays that support integrin-mediated adhesion and function as plasmonic nanostoves to physically block and orient the formation of new adhesion sites.

\section{RESULT AND DISCUSSION}

Although many techniques are available for the fabrication of nanoparticlebased devices, they all have significant shortcomings in using benchtop facilities, minimizing the number of preparation steps and controlling independently the device structural parameters. Among various advanced lithographic techniques, block-copolymer micellar lithography $(B C M L)$ has been recognized as a straightforward method for generating a wide variety of periodic nanostructures with a long-range order. ${ }^{18,19}$ Although interparticle distance can be adjusted from 25 to $250 \mathrm{~nm}$, control over particle diameter is restricted to sizes below $10 \mathrm{~nm}$. Here, we used quasi-hexagonally organized gold nanoarrays with a separation distance of about $90 \mathrm{~nm}$ and a particle diameter of $7 \mathrm{~nm}$. As such nanostructured surfaces do not exhibit an intense absorption band for effective photothermal effects, an additional step is 
needed to grow larger gold particles. Hydroxylamine seeding of colloidal gold $^{20}$ was shown to be applicable to gold nanoarrays upon functionalizing the substrate with alkyl chains for preventing particles to spontaneously lift-off during size enlargement. ${ }^{18}$ To avoid this laborious substrate modification, we directly immersed glass-supported gold nanoarrays into an aqueous solution of $\mathrm{Au}^{3+}$ and tested for the first time ethanolamine as reducing agent. Since alkylamines can mediate the generation of gold nanoparticles while acting as dual capping and reducing agents, ${ }^{21}$ we chose ethanolamine because of its water solubility. As displayed in Figure 1, we successfully applied ethanolamine seeding of colloidal gold to nanoarrays for which the particle diameter homogeneously increased from 7 to $32 \mathrm{~nm}$ in 20 minutes and the plasmon band intensity centered at $530 \mathrm{~nm}$ became significantly stronger.

To make these nanostructures compatible with cell experiments, we first passivated the glass background with poly(ethylene glycol) (PEG) and functionalized gold with cysteine-terminated linear RGD. ${ }^{22}$ RGD is a conserved motif found in several matrix proteins, such as fibronectin. RGD epitopes are bound by transmembrane receptors of the integrin family, which mediate adhesion and physically connect the cytoskeleton to the $\mathrm{ECM}^{23,24}$ In tissue culture, cells migrate on extended surfaces in random directions. To orient their behavior, it is possible to confine cells onto defined areas by using micropatterned substrates coated with ECM proteins. For instance, cell migration on thin fibronectin-coated lines was shown to be faster and more persistent similarly as in vivo-like situations. ${ }^{25}$ To apply this concept to our setup, we decided to structure gold nanoparticle distribution onto $10 \mu \mathrm{m}$-wide lines for confining cell adhesion into one dimension. 
To prepare micropatterned gold nanoarrays, BCML can be combined to other lithography techniques. For instance, electron-beam lithography can directly fix gold-loaded micelles onto substrates via a serial exposure process. ${ }^{26}$ Despite a patterning precision of $100 \mathrm{~nm}$, this method necessitates a scanning electron microscope and long processing rates. More commonly used, parallel procedures are more effective to fabricate simultaneously patterns with sizes that remain larger than one micrometer. ${ }^{27-29}$ However, these advanced protocols still hinder the high sample throughput necessary for biological screening experiments. In an effort to work with affordable facilities and minimize the number of processing steps, we used the simplicity of a serial approach in a parallel fashion by using deep UV illumination onto gold-loaded micellar monolayers. With a wavelength of $185 \mathrm{~nm}$, deep UV lithography can directly photoconvert chemical groups without using photosensitizers and cross-linkers. ${ }^{30}$ Here, we successfully prepared micropatterned gold nanoparticles by illuminating micellar monolayers with deep UV through a photomask and transferring the substrate in toluene to liftoff non-irradiated micelles (Figure 2). As revealed by X-ray photoelectron spectroscopy (Figure S1), the immobilization of micelles occurred upon partially oxidizing their polystyrene shells with deep UV, which enhances their affinity to the glass substrate and reduces their solubility in toluene. As displayed in Figure 2, this approach can rapidly micropattern gold nanoparticles with various geometries and feature sizes of about $500 \mathrm{~nm}$, while conserving their quasi-hexagonal organization.

Next, we seeded mouse fibroblasts onto $10 \mu \mathrm{m}$-wide lines upon passivating the glass background and biofunctionalizing gold nanoparticles of $35 \mathrm{~nm}$ in 
diameter. Similarly as on fibronectin-coated lines, fibroblasts selectively adhered to the linear RGD-functionalized gold nanoarrays and adopted a polarized shape to initiate migration without modifying the structure of gold nanoarrays (Figure 3 and S2). Upon staining filamentous actin (F-actin) and paxillin, an adaptor protein that connects actin cytoskeleton to the cytoplasmic tail of integrins, cells could assemble similar intracellular architectures on both types of substrates, as revealed by confocal fluorescence microscopy (Figure $3)$.

Besides confining the migration path of fibroblasts, micropatterned gold nanoarrays have the ability to generate heat when illuminated with a laser beam at $\lambda=532 \mathrm{~nm}$, close to the plasmon resonance wavelength. In contrast to electrically-heated microwires, the use of thermoplasmonic structures provide more spatial freedom, as control over thermal variations does not only depend on pattern geometry but also on laser position. By using quantitative optical phase analysis that enables to image heat profiles, ${ }^{31}$ we found that temperature increases proportionally with laser power. Although the laser beam displays a homogeneous power density, we measured thermal variations of $12.8,18.7$ and $24.2 \mathrm{~K}$ at the beam center and $7.5,13$ and $16.7 \mathrm{~K}$ at its periphery for 2, 3 and $4 \mathrm{~mW}$ output powers, respectively (Figure 4). The temperature variations follow a parabolic profile within the illumination area and it decreases in $1 / R$ outside the beam. ${ }^{31}$ Since many cellular components absorb visible light, we decided to overcome phototoxicity by benefiting from the temperature variations taking place outside the illumination area to manipulate cells. 
Here, we tested the ability of local heat to interfere with cell adhesion by simultaneously blocking and guiding protrusive activity during spreading and migration. For this purpose, we first modified an epifluorescence microscope by replacing the excitation lamp with a green diode laser (Figure S3). Upon seeding fibroblasts, we first monitored cell spreading with live-cell imaging in different conditions (Figure 5a,b,c, Movie 1). Without laser, the cell spread equally in both directions for 30 minutes and finally polarized to initiate migration (Figure 5a). By illuminating cell surrounding with a laser beam of about $3 \mathrm{~mW}$, the generation of local heat blocked the formation of adhesion sites in one direction and allowed protrusive activity in the opposite one, leading the cell to polarize earlier and initiate migration faster (Figure 5b). As a control experiment, we immobilized RGD epitopes onto PEG-coated glass ${ }^{30}$ to prevent heat generation (Figure $5 \mathrm{c}$ ). In these conditions, the cell entered the laser beam and died within less than twenty minutes due to phototoxicity, confirming the ability of local heat to restrain cells to adhere. As a second example of cell manipulation, migrating cells could also be stopped for up to 30 minutes by local heat, acting similarly as a wall (Figure $5 \mathrm{~d}, \mathrm{e}$, Movie 2). Upon turning the laser illumination off, cells instantly reinitiated migration in the same direction, which emphasizes the reversible character of thermoplasmonic manipulations. In both examples, it is interesting to note the ability of cells to adapt their behavior depending on local temperature. Blocking protrusive activity may implicate the inability of integrins to tightly bind to RGD epitopes in a warmer environment. According to Figure 4, cell periphery experiences a local temperature of about $44{ }^{\circ} \mathrm{C}$ when localized at $2.5 \mu \mathrm{m}$ away from the beam border. Although such a temperature 
corresponds to an extreme physiological condition, cells neither died nor lost their ability to migrate persistently.

\section{CONCLUSION}

In the present study, we report a simple and cost-effective protocol to micropattern thermoplasmonic nanoarrays by combining deep UV and micellar lithography. Being reversible and not restricted spatiotemporally, the generation of photothermal gradients in the vicinity of cells interferes microlocally with cell adhesion and allows both guiding and blocking cell migration in a defined microenvironment. As future challenges, it would be crucial to extend the use of deep UV-assisted micellar nanolithography for designing gold structures that absorb wavelengths within the tissue transparency window, such as near-infrared. By overcoming cellular phototoxicity and fluorophore photobleaching, thermoplasmonic manipulation could be performed while analyzing live-cell spreading, migration and detachment by fluorescence microscopy. Moreover, this approach could be applied to spatiotemporally manipulate the affinities of other cell surface receptors to their respective ligands, which would enable to further dissect reciprocal signaling circuits between cells and their environment. 


\section{EXPERIMENTAL METHODS}

Fabrication of extended gold nanoarrays. In a typical synthesis, polystyrene(1056)-blockpoly(2-vinylpyridine)(495) $\left(\mathrm{PS}_{1056}-b-\mathrm{P}_{2} \mathrm{VP}_{495}\right)$ from Polymer Source Inc. was dissolved at room temperature in anhydrous toluene (Sigma-Aldrich) with a concentration of $5 \mathrm{mg} / \mathrm{mL}$ and stirred for 2 days. The quantity of gold precursor was calculated relative to the number of P2VP units ( $N_{P 2 V P}$ ) with a loading parameter (L) equal to 0.5, i.e., 1 molecule of $\mathrm{HAuCl}_{4}$ for 2 P2VP monomers. Hydrogen tetrachloroaurate (III) trihydrate $\left(\mathrm{HAuCl}_{4} \cdot 3 \mathrm{H}_{2} \mathrm{O}\right.$, Sigma-Aldrich) was added to the polymer solution and stirred for 2 days in a sealed glass vessel. Glass cover slips (Carl Roth) were cleaned in a piranha solution for at least 5 hours and were extensively rinsed with MilliQ water and dried under a stream of nitrogen. Gold-loaded micellar monolayers were prepared by dip-coating a glass coverslip into the previously prepared solutions with a constant velocity equal to $24 \mathrm{~mm} \cdot \mathrm{min}^{-1}$. To remove the organic template and form inorganic nanoparticles, the dip-coated glass slides were exposed to oxygen plasma (150 W, $0.15 \mathrm{mbar}, 45 \mathrm{~min}$, PVA TEPLA 100 Plasma System). Scanning electron measurements were performed with a Dual Beam ${ }^{\mathrm{TM}}$ (FIB/SEM) instrument (Quanta 3D FEG, FEI, Hillsboro).

Fabrication of micropatterned gold nanoarrays. This protocol is schematically described in Figure 2. Briefly, a glass coverslip coated with a gold-loaded micellar monolayer was immobilized with vacuum onto a mask holder and then sandwiched with a photomask made of a patterned chromium layer supported on UV-transparent quartz (ML\&C, Jena). The system was then exposed to deep UV light using a low-pressure mercury lamp (Heraeus Noblelight $\mathrm{GmbH}, \mathrm{NIQ} 60 / 35 \mathrm{XL}$ long-life lamp, quartz tube, $60 \mathrm{~W}$ ) at $5 \mathrm{~cm}$ distance for $7 \mathrm{~min}$. The micellar monolayer was then incubated for 30 minutes in toluene to wash non-irradiated micelles away and dried with the hood airflow. The enlargement of immobilized gold nanoparticles was performed by incubating the substrate for several minutes in $10 \mathrm{~mL}$ of an aqueous solution containing ethanolamine $(2 \mathrm{mM})$ and $\mathrm{KAuCl}_{4}(0.1 \mathrm{wt} \%)$.

Surface functionalization. Gold nanoarrays supported on glass coverslips were incubated in water with $0.25 \mathrm{mg} / \mathrm{mL}$ of PLL-g-PEG (SuSoS) for 2 hours. The substrates were rinsed with water, dried with nitrogen and incubated for 5 hours in a $50 \mu \mathrm{M}$ aqueous solution of 
fibronectin-mimicking polypeptide, N-terminated $\mathrm{C}-\mathrm{L}_{3} \mathrm{G}_{3} \mathrm{PHRSN}-\mathrm{G}_{6}-\mathrm{RGDS}^{32}$ Finally, the substrates were rinsed and incubated overnight in water before plating fibroblasts.

Thermal imaging of micropatterned gold nanoarrays ${ }^{31}$. Temperature measurements were performed by quadriwave shearing interferometry (TIQSI), a recent thermal imaging technique based on thermal-induced variation of the refractive index of the liquid environment. This approach is particularly simple since it is label-free and it allows rapidly and quantitatively mapping a temperature distribution with an accuracy of less than $1 \mathrm{~K}$. Due to the modification of the refractive index in the heated region, the optical wavefront of an incident light experiences a distortion that is imaged using a Hartmann grating in front of a CCD camera. The interferogram recorded by the CCD camera is then numerically processed to retrieve the actual temperature distribution.

Setup for thermoplasmonic manipulation. In order to microlocally generate heat while performing live-cell imaging, we equipped a Zeiss Axiovert 40 CFL microscope (Carl Zeiss, Jena, Germany) with a cell culture chamber $\left(37^{\circ} \mathrm{C}, 5 \% \mathrm{CO}_{2}\right)$, a green laser (Laserglow, Hercules 250, $\lambda=532 \mathrm{~nm}$ ), a beam-splitter, a band pass filter and a camera (Canon, EOS 450D), as depicted in Supplementary Fig. S4. For cell manipulation experiments, approximately $1.10^{6}$ cells in DMEM containing $0.5 \%$ FBS were seeded onto functionalized micropatterned gold nanoarrays. Thermoplasmonic experiments were conducted within the following 10 hours to avoid the contribution of ECM secretion.

Immunofluorescence. After three hours in culture, fibroblasts were fixed with 2\% PFA, permeabilised with $0.1 \%$ Triton $\mathrm{X}-100$ and incubated with primary and secondary antibodies.

Paxillin was stained with anti-paxillin (mouse monoclonal; Transduction Laboratories), F-actin with phalloidin (Molecular Probes) and nuclei with DAPI. Secondary antibodies used for immunofluorescence were from Invitrogen (Alexa488, Alexa647). The fluorescent images were collected by laser scanning confocal microscopy (TCS SP5; Leica) with a 63x oil objective and Leica confocal software.

Acknowledgments. We thank Reinhard Fässler for support and Herbert Schiller for careful reading of the manuscript. The $7^{\text {th }}$ Framework Program of the European Community (FP7) through the Marie Curie Action for career development (IEF - "Multi-PGNAs") and the Max Planck Society financially supported this work. 
Supporting Information Available: Supplementary methods; X-ray photoelectron spectroscopy analysis; additional low and high-magnification SEM images and schematics of microscope setup used for thermoplasmonic manipulation. This material is available free of charge via the Internet at http://pubs.acs.org.

\section{REFERENCES AND NOTES}

1. Vicente-Manzanares, M.; Ma, X.; Adelstein, R. S.; Horwitz, A. R., Non-muscle myosin II takes centre stage in cell adhesion and migration. Nat Rev Mol Cell Biol 2009, 10, 778-790.

2. Fenteany, G.; Zhu, S., Small-molecule inhibitors of actin dynamics and cell motility. Curr Top Med Chem 2003, 3, 593-616.

3. Collier, J.; Mrksich, M., Engineering a biospecific communication pathway between cells and electrodes. Proc Natl Acad Sci USA 2006, 103, 2021-2025.

4. Nakanishi, J.; Kikuchi, Y.; Inoue, S.; Yamaguchi, K.; Takarada, T.; Maeda, M., Spatiotemporal control of migration of single cells on a photoactivatable cell microarray. J Am Chem Soc 2007, 129, 6694-6695.

5. Wildt, B.; Wirtz, D.; Searson, P. C., Programmed subcellular release for studying the dynamics of cell detachment. Nat Methods 2009, 6, 211-213.

6. Jiang, X.; Bruzewicz, D. A.; Wong, A. P.; Piel, M.; Whitesides, G. M., Directing cell migration with asymmetric micropatterns. Proc Natl Acad Sci USA 2005, 102, 975-8.

7. Rolli, C. G.; Nakayama, H.; Yamaguchi, K.; Spatz, J. P.; Kemkemer, R.; Nakanishi, J., Switchable adhesive substrates: Revealing geometry dependence in collective cell behavior. Biomaterials 2012, 33, 2409-2418.

8. Robertus, J.; Browne, W. R.; Feringa, B. L., Dynamic control over cell adhesive properties using molecular-based surface engineering strategies. Chem Soc Rev 2010, 39, 354.

9. Nagase, K.; Kobayashi, J.; Okano, T., Temperature-responsive intelligent interfaces for biomolecular separation and cell sheet engineering. J R Soc Interface 2009, 6, S293-S309.

10. Ginet, P.; Montagne, K.; Akiyama, S.; Rajabpour, A.; Taniguchi, A.; Fujii, T.; Sakai, Y.; Kim, B.; Fourmy, D.; Volz, S., Towards single cell heat shock response by accurate control on thermal confinement with an on-chip microwire electrode. Lab Chip 2011, 11, 1513.

11. Baffou, G.; Quidant, R., Thermo-plasmonics: using metallic nanostructures as nano-sources of heat. Laser \& Photon. Rev. 2012, early view.

12. Liu, G. L.; Kim, J.; Lu, Y.; Lee, L. P., Optofluidic control using photothermal nanoparticles. Nat Mater 2006, 5, 27-32.

13. Donner, J. S.; Baffou, G.; McCloskey, D.; Quidant, R., Plasmon-Assisted Optofluidics. Acs Nano 2011, 5, 5457-5462.

14. Stehr, J.; Hrelescu, C.; Sperling, R. A.; Raschke, G.; Wunderlich, M.; Nichtl, A.; Heindl, D.; Kurzinger, K.; Parak, W. J.; Klar, T. A.; Feldmann, J., Gold NanoStoves for microsecond DNA melting analysis. Nano Lett. 2008, 8, 619-623.

15. Hirsch, L.; Stafford, R.; Bankson, J.; Sershen, S.; Rivera, B.; Price, R.; Hazle, J.; Halas, N.; West, J., Nanoshell-mediated near-infrared thermal therapy of tumors under magnetic resonance guidance. Proc Natl Acad Sci USA 2003, 100, 13549-13554.

16. El-Sayed, I.; Huang, X.; El-Sayed, M., Surface plasmon resonance scattering and absorption of anti-EGFR antibody conjugated gold nanoparticles in cancer diagnostics: Applications in oral cancer. Nano Lett. 2005, 5, 829-834.

17. Huschka, R.; Neumann, O.; Barhoumi, A.; Halas, N. J., Visualizing Light-Triggered Release of Molecules Inside Living Cells. Nano Lett. 2010, 10, 4117-4122.

18. Lohmueller, T.; Aydin, D.; Schwieder, M.; Morhard, C.; Louban, I.; Pacholski, C.; Spatz, J. P., Nanopatterning by block copolymer micelle nanolithography and bioinspired applications. Biointerphases 2011, 6, MR1-MR12.

19. Polleux, J.; Rasp, M.; Louban, I.; Plath, N.; Feldhoff, A.; Spatz, J. P., Benzyl Alcohol and Block Copolymer Micellar Lithography: A Versatile Route to Assembling Gold and in Situ Generated Titania Nanoparticles into Uniform Binary Nanoarrays. Acs Nano 2011, 5, 6355-6364.

20. Brown, K.; Lyon, L.; Fox, A.; Reiss, B.; Natan, M., Hydroxylamine seeding of colloidal au nanoparticles. 3. Controlled formation of conductive Au films. Chem Mater 2000, 12, 314-323.

21. Aslam, M.; Fu, L.; Su, M.; Vijayamohanan, K.; Dravid, V. P., Novel one-step synthesis of amine-stabilized aqueous colloidal gold nanoparticles. J. Mater. Chem. 2004, 14, 1795. 
22. Arnold, M.; Cavalcanti-Adam, E.; Glass, R.; Blummel, J.; Eck, W.; Kantlehner, M.; Kessler, H.; Spatz, J., Activation of integrin function by nanopatterned adhesive interfaces. Chemphyschem 2004, 5, 383-388.

23. Polleux, J., Bioactive Surfaces. Interfacing Cell Surface Receptors to Hybrid Nanopatterned Surfaces: A Molecular Approach for Dissecting the Adhesion Machinery; Börner H. G., Lutz J. F., Eds.; Springer-Verlag: Heidelberg Berlin, 2011, 240, 79-102.

24. Takahashi, S.; Leiss, M.; Moser, M.; Ohashi, T.; Kitao, T.; Heckmann, D.; Pfeifer, A.; Kessler, H.; Takagi, J.; Erickson, H. P.; Fassler, R., The RGD motif in fibronectin is essential for development but dispensable for fibril assembly. J Cell Biol 2007, 178, 167-178.

25. Doyle, A. D.; Wang, F. W.; Matsumoto, K.; Yamada, K. M., One-dimensional topography underlies three-dimensional fibrillar cell migration. $J$ Cell Biol 2009, 184, 481-90.

26. Spatz, J.; Chan, V.; Mossmer, S.; Kamm, F.; Plettl, A.; Ziemann, P.; Moller, M., A combined top-down/bottom-up approach to the microscopic localization of metallic nanodots. Adv. Mater. 2002, $14,1827-1832$.

27. Aydin, D.; Schwieder, M.; Louban, I.; Knoppe, S.; Ulmer, J.; Haas, T. L.; Walczak, H.; Spatz, J. P., Micro-Nanostructured Protein Arrays: A Tool for Geometrically Controlled Ligand Presentation. Small 2009, 5, 1014-1018.

28. Chen, J.; Mela, P.; Moeller, M.; Lensen, M. C., Microcontact Deprinting: A Technique to Pattern Gold Nanoparticles. Acs Nano 2009, 3, 1451-1456.

29. Gorzolnik, B.; Mela, P.; Moeller, M., Nano-structured micropatterns by combination of block copolymer self-assembly and UV photolithography. Nanotechnology 2006, 17, 5027-5032.

30. Azioune, A.; Storch, M.; Bornens, M.; Thery, M.; Piel, M., Simple and rapid process for single cell micro-patterning. Lab Chip 2009, 9, 1640-1642.

31. Baffou, G.; Bon, P.; Savatier, J.; Polleux, J.; Zhu, M.; Merlin, M.; Rigneault, H.; Monneret, S., Thermal Imaging of Nanostructures by Quantitative Optical Phase Analysis. Acs Nano 2012, 6, 2452-2458.

32. Ebara, M.; Yamato, M.; Aoyagi, T.; Kikuchi, A.; Sakai, K.; Okano, T., A Novel Approach to Observing Synergy Effects of PHSRN on Integrin-RGD Binding Using Intelligent Surfaces. Adv. Mater. 2008, 20, 3034-3038. 

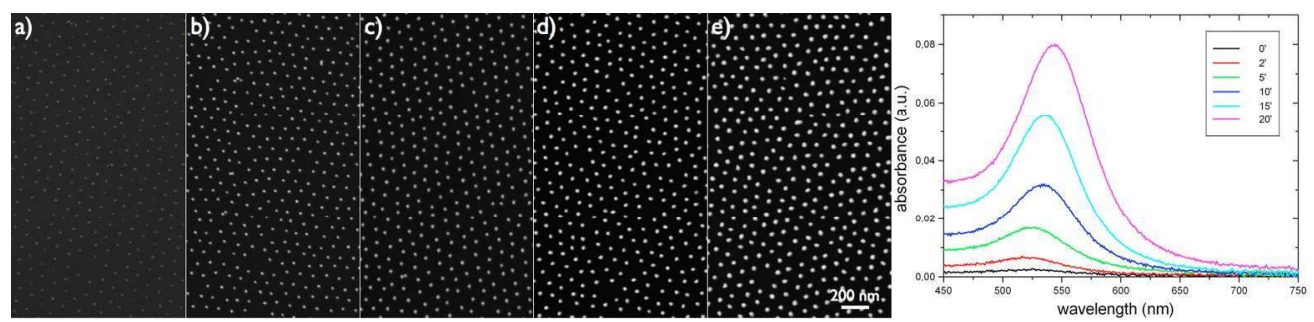

Ethanolamine-assisted growth of gold nanoparticles supported on glass coverslips. SEM images of gold nanoarrays after immersion for (a) 0, (b) 5, (c) 10, (d) 15 and (e) 20 minutes in an aqueous solution of ethanolamine (2mM) and $\mathrm{KAuCl} 4(0.1 \mathrm{wt} \%)$. (f) Absorption spectra of the different gold nanoarrays upon rinsing with water and drying with $\mathrm{N} 2$. $677 \times 159 \mathrm{~mm}(72 \times 72 \mathrm{DPI})$ 
a)

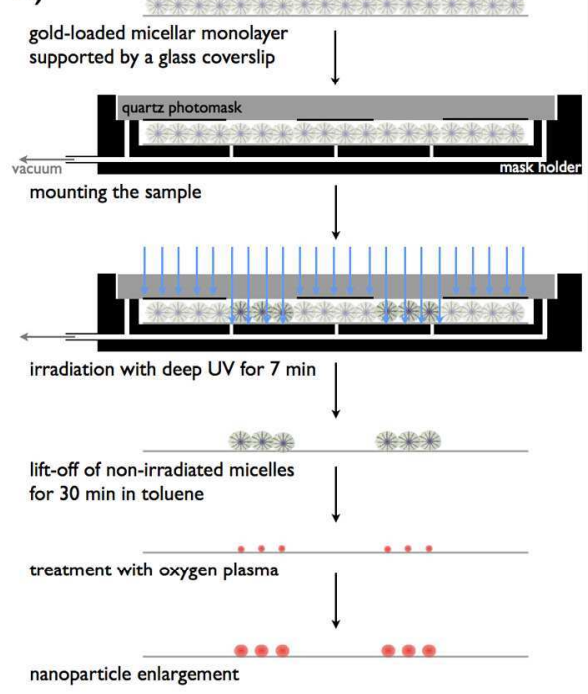

b)
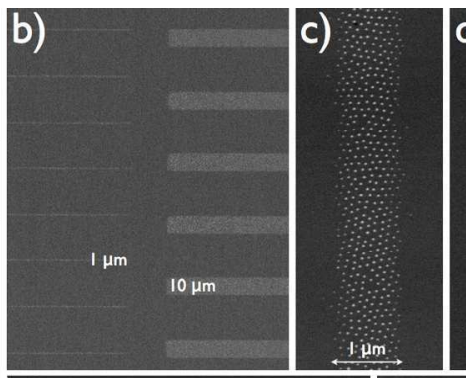

d)

e)

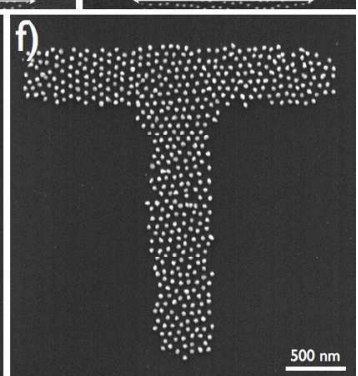

Combining deep UV and micellar lithography to micropattern thermoplasmonic gold nanoarrays. (a) Schematics displaying the successive steps necessary to fabricate micropatterned gold nanoparticles. (b) Low-magnification SEM image of nanostructured lines with a width of 1 and $10 \mu \mathrm{m}$. (c), (d), (e), and (f), display high-resolution SEM images of micropatterned gold nanoarrays of various shapes and dimensions. $677 \times 381 \mathrm{~mm}(72 \times 72 \mathrm{DPI})$ 

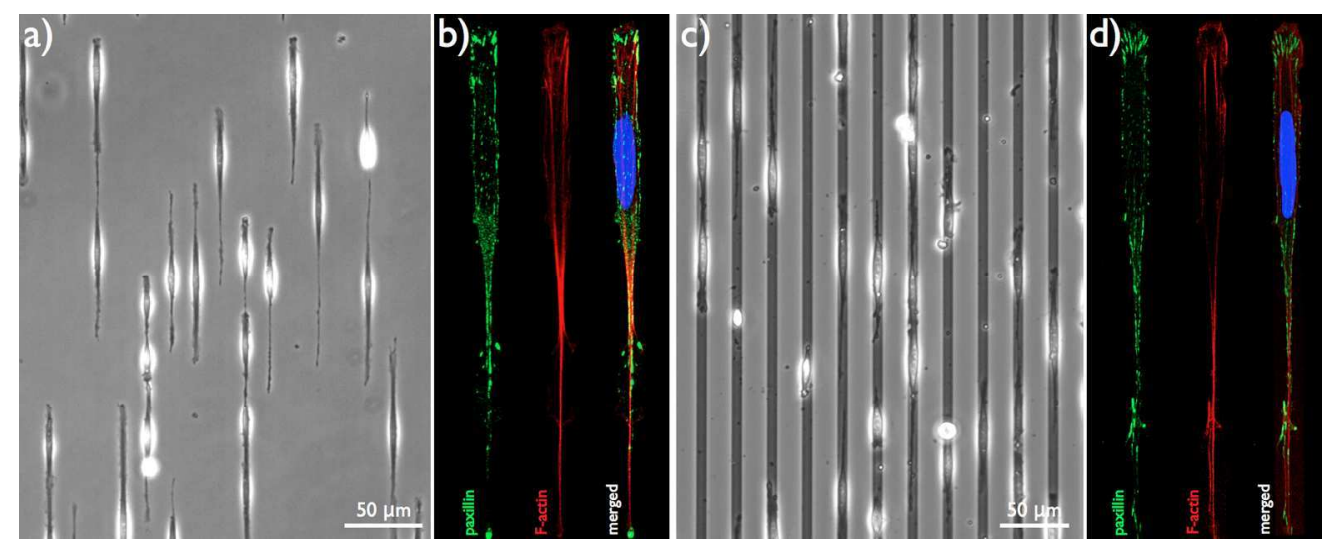

Cell adhesion on FN- and RGD-coated $10 \mu \mathrm{m}$-wide lines occurs similarly. Phase contrast microscopy images of mouse fibroblasts migrating onto (a) FN-coated glass and (c) RGD-functionalized gold nanoparticles of 35 $\mathrm{nm}$ in diameter separated by $90 \mathrm{~nm}$. Confocal fluorescence microscopy images of fixed cells stained with GFP-labeled paxillin and red-labeled F-actin displaying adhesive sites and cytoskeleton of a cell migrating onto (b) FN-coated glass, and (d) RGD-coated gold nanoparticles. $677 \times 273 \mathrm{~mm}(72 \times 72 \mathrm{DPI})$ 


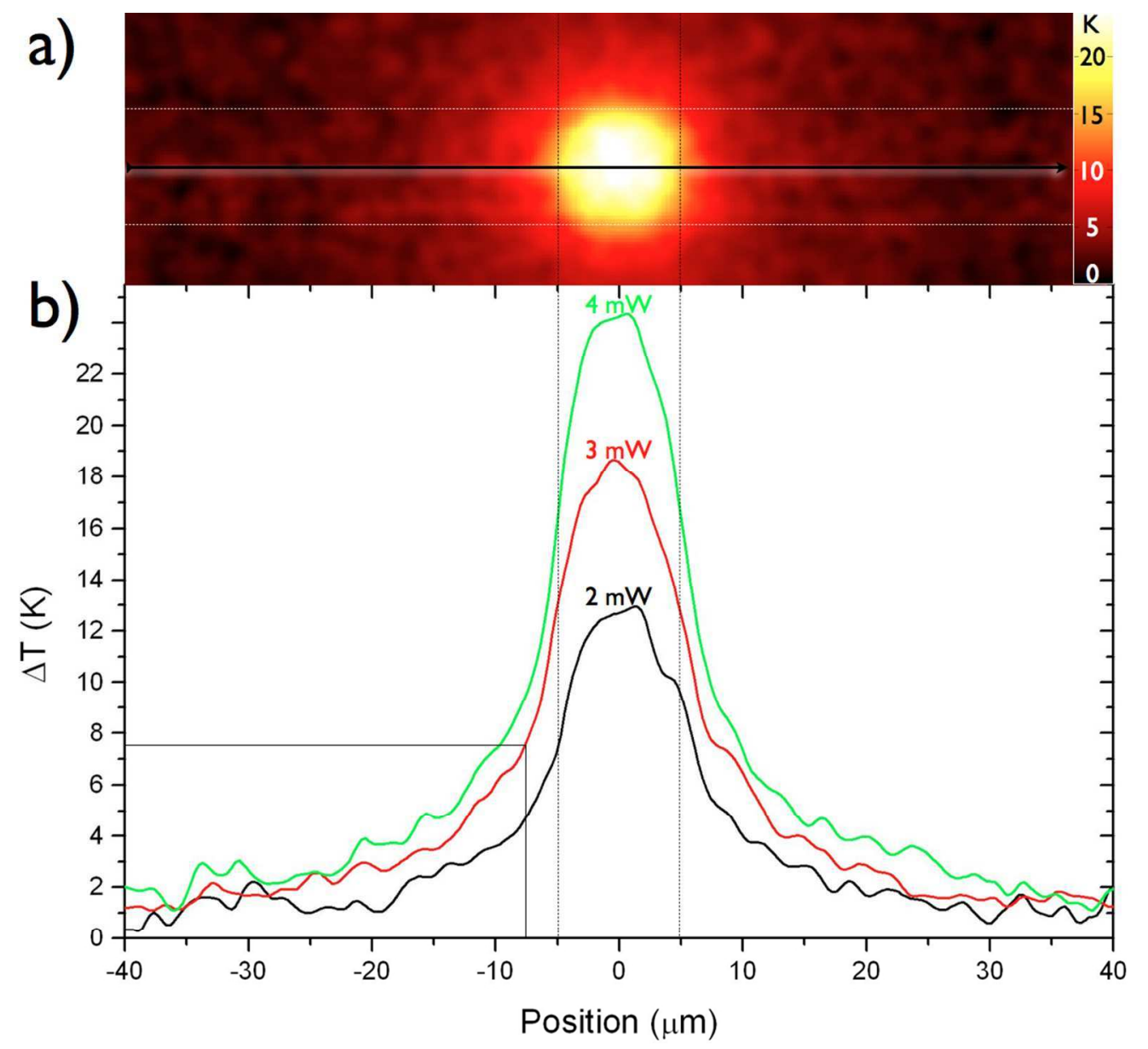

Photothermal effects of a quasi-hexagonal lattice of spherical gold nanoparticles under laser illumination at $\lambda=532 \mathrm{~nm}$. The measurements were performed on a gold nanoarrays made of $35 \mathrm{~nm}$ particles with an interdistance of $90 \mathrm{~nm}$ and irradiated by a beam of $10 \mu \mathrm{m}$ in diameter at powers of 2,3 and $4 \mathrm{~mW}$. (a) Temperature distribution exposed to a laser power of $4 \mathrm{~mW}$ and (b) temperature profile obtained from the linescan (black arrow in (a)). The white dashed lines indicate the borders of the $10 \mu \mathrm{m}$-wide lines made of gold particles.

\section{$423 \times 392 \mathrm{~mm}(72 \times 72$ DPI $)$}


Heat-assisted manipulation of cell adhesion during spreading and migration: Guiding cell migration during spreading. Phase contrast images of cells spreading onto RGD-coated nanoparticles of $35 \mathrm{~nm}$ in diameter (a) without and (b) with laser illumination of $3 \mathrm{~mW}$ for 40 minutes. (c) Cell spreading onto RGD-coated glass coverslip under the same irradiation conditions as in (b). Blocking cell adhesion during migration. Phase contrast images of cells migrating onto RGD-coated particles (d) without and (e) with laser illumination of 3 $\mathrm{mW}$ for 30 minutes. Each image has a time interval of 10 minutes $722 \times 524 \mathrm{~mm}(72 \times 72 \mathrm{DPI})$ 